\title{
TORUS CANNOT COLLAPSE TO A SEGMENT
}

\author{
MIKHAIL G. KATZ
}

\begin{abstract}
In earlier work, we analyzed the impossibility of codimension-one collapse for surfaces of negative Euler characteristic under the condition of a lower bound for the Gaussian curvature. Here we show that, under similar conditions, the torus cannot collapse to a segment. Unlike the torus, the Klein bottle can collapse to a segment; we show that in such a situation, the loops in a short basis for homology must stay a uniform distance apart.
\end{abstract}

\section{IntRoduCtion}

In earlier work [6], we analyzed the impossibility of codimension-one collapse for surfaces of negative Euler characteristic, if Gaussian curvature is bounded from below and the diameter from above. Here we show that, under similar conditions, the torus cannot collapse to a segment, answering a question posed at MO [1]. For a general framework for collapse of Riemannian manifolds, see [11, [12], [3, Section 8.25].

Recall that an embedding $N \subseteq M$ of Riemannian manifolds is called strongly isometric if the ambient distance and the intrinsic distance in $N$ coincide.

Lemma 1.1. Let $M$ be a closed surface with a Riemannian metric. Consider a homology basis

$$
\left[\alpha_{1}\right], \ldots,\left[\alpha_{r}\right] \in H_{1}\left(M ; \mathbb{Z}_{2}\right)
$$

such that $\left|\alpha_{1}\right| \leq \ldots \leq\left|\alpha_{r}\right|$ and the sum of the lengths of the loops $\alpha_{i}$ is minimal. Then each of the inclusions $\alpha_{1} \subseteq M, \ldots, \alpha_{r} \subseteq M$ is strongly isometric.

Proof. We argue by contradiction. Suppose that an inclusion $\alpha_{i} \subseteq M$ is not strongly isometric. Then there are points $x, y \in \alpha_{i}$ partitioning $\alpha_{i}$ into $\operatorname{arcs} \alpha_{i}^{\prime}, \alpha_{i}^{\prime \prime}$ such that a minimizing path $\eta$ between $x$ and $y$ is shorter than either arc. Consider the loops $\alpha_{i}^{\prime} \cup \eta$ and $\alpha_{i}^{\prime \prime} \cup \eta$ obtained as union of paths. These loops are shorter than $\alpha_{i}$. In the homology group, we have the relation

$$
\left[\alpha_{i}^{\prime} \cup \eta\right]+\left[\alpha_{i}^{\prime \prime} \cup \eta\right]=\left[\alpha_{1}\right] \in H_{1}\left(M ; \mathbb{Z}_{2}\right),
$$


where orientations can be ignored since we are working with $\mathbb{Z}_{2}$-coefficients. Exactly one of the new loops, say $\alpha_{i}^{\prime} \cup \eta$, lies outside the hyperplane spanned by the classes of $\left(\alpha_{1}, \ldots, \alpha_{i-1}, \alpha_{i+1}, \ldots, \alpha_{r}\right)$. Since we are working with $\mathbb{Z}_{2}$-coefficients, the family of loops

$$
\left(\alpha_{1}, \ldots, \alpha_{i-1}, \alpha_{i}^{\prime} \cup \eta, \alpha_{i+1}, \ldots, \alpha_{r}\right)
$$

is a basis for homology. By construction, the sum of the lengths of this new family of loops is smaller than the sum of the lengths of the original family (1.1), contradicting the definition of that family and proving the lemma.

Corollary 1.2. When $M=T^{2}$ is the 2-torus, the loops $\alpha=\alpha_{1}$ and $\beta=\alpha_{2}$ represent elements of a basis of $H_{1}\left(T^{2} ; \mathbb{Z}\right)$ and meet at a single point.

Proof. By definition,

$$
|\alpha| \leq|\beta|
$$

Since the classes $[\alpha],[\beta] \in H_{1}\left(T^{2} ; \mathbb{Z}_{2}\right)$ are distinct, they have nonzero algebraic intersection and therefore nontrivial geometric intersection. To prove that $\alpha$ and $\beta$ also represent a basis of $H_{1}\left(T^{2} ; \mathbb{Z}\right)$, it suffices to show that the intersection $\alpha \cap \beta$ consists of a single point.

We will argue by contradiction. Suppose there are two distinct points $x, y$ in $\alpha \cap \beta$. Let $\alpha_{0}$ be a shortest arc of $\alpha$ connecting $x$ and $y$, and $\beta_{0}$ a shortest arc of $\beta$ connecting $x$ and $y$.

Suppose $\alpha_{0} \sim \beta_{0}$ (i.e., the union $\alpha_{0} \cup \beta_{0}$ is $\mathbb{Z}_{2}$-nullhomologous). Then either $\alpha$ or $\beta$ can be shortened by replacing its arc between $x$ and $y$ by the corresponding arc of the other loop, and rounding off the corners. This contradicts the minimality of the basis $[\alpha],[\beta]$.

Hence $\alpha_{0} \cup \beta_{0}$ represents a nonzero class in $H_{1}\left(T^{2} ; \mathbb{Z}_{2}\right)$. By construction $\left|\alpha_{0} \cup \beta_{0}\right| \leq|\beta|$. Let $\delta$ be a minimizing loop in the class $\left[\alpha_{0} \cup \beta_{0}\right] \in$ $H_{1}\left(T^{2} ; \mathbb{Z}_{0}\right)$. Then $|\delta|<|\beta|$. Now (1.2) implies that

$$
\delta \in[\alpha]=\left[\alpha_{0} \cup \beta_{0}\right],
$$

and therefore $|\delta|=|\alpha|$.

Consider the complementary subarcs $\alpha_{1}=\alpha \backslash \alpha_{0}$ and $\beta_{1}=\beta \backslash \beta_{0}$. By (1.3) we have $\alpha_{1} \sim \beta_{0}$. If $\left|\beta_{0}\right|<\left|\alpha_{1}\right|$ then $\alpha$ can be shortened to $\alpha_{0} \cup \beta_{0}$, contradicting the minimality of the loop $\alpha$ in its $\mathbb{Z}_{2}$-homology class; if $\left|\beta_{0}\right|>\left|\alpha_{1}\right|$ then $\beta$ can be shortened to $\alpha_{1} \cup \beta_{1}$, contradicting the minimality of the loop $\beta$ in its $\mathbb{Z}_{2}$-homology class (the case of equality is treated by rounding off corners as before). The contradiction proves that $\alpha$ and $\beta$ generate a basis for integer homology.

Note that the conclusion of Corollary 1.2 may fail for the Klein bottle; cf. Example 5.1. 
Definition 1.3. Let $p=\alpha \cap \beta \in T^{2}$ be the intersection point of the two loops.

Thus, the loops $\alpha$ and $\beta$ represent generators of $\pi_{1}\left(T^{2}, p\right) \simeq \mathbb{Z}^{2}$.

Definition 1.4. Choosing a parametrisation for each of the loops based at $p$, we form the commutator loop $\alpha \circ \beta \circ \alpha^{-1} \circ \beta^{-1}$ based at $p$.

\section{Circle noncollapse}

Lemma 2.1. Suppose there exists a metric d on the disjoint union of a circle $S$ of length $L$ and a segment $I$ such that both $S$ and $I$ are strongly isometrically embedded. Assume that $S$ is contained in a $\nu$ neighborhood of $I$. Then $\nu>\frac{L}{16}$.

Proof. Let $a, b \in S$ be two antipodal points on $S$. Let $J_{1}$ and $J_{2}$ be the two arcs of $S$ connecting $a$ and $b$. Clearly $\left|J_{1}\right|=\left|J_{2}\right|=\frac{L}{2}$. Both $J_{1}$ and $J_{2}$ are strongly isometrically embedded in $S \cup I$. Choose $a^{\prime}, b^{\prime} \in I$ such that $d\left(a, a^{\prime}\right)<\nu$ and $d\left(b, b^{\prime}\right)<\nu$. Clearly $\left|d\left(a^{\prime}, b^{\prime}\right)-\frac{L}{2}\right|<2 \nu$. Let $c_{1}$ and $c_{2}$ be the midpoints of the arcs $J_{1}$ and $J_{2}$, respectively. One can find points $c_{1}^{\prime}, c_{2}^{\prime} \in I$ such that $d\left(c_{1}, c_{1}^{\prime}\right)<\nu$ and $d\left(c_{2}, c_{2}^{\prime}\right)<\nu$. We have

$$
\left|d\left(a^{\prime}, c_{i}^{\prime}\right)-\frac{L}{4}\right|<2 \nu,\left|d\left(b^{\prime}, c_{i}^{\prime}\right)-\frac{L}{4}\right|<2 \nu \text { for } i=1,2 .
$$

It follows that

$$
\left|d\left(a^{\prime}, c_{i}^{\prime}\right)-\frac{1}{2} d\left(a^{\prime}, b^{\prime}\right)\right|<3 \nu,\left|d\left(b^{\prime}, c_{i}^{\prime}\right)-\frac{1}{2} d\left(a^{\prime}, b^{\prime}\right)\right|<3 \nu \text { for }, i=1,2 .
$$

Let $c^{\prime}$ be the midpoint of the segment $\left[a^{\prime}, b^{\prime}\right]$. Then $d\left(c^{\prime}, c_{i}^{\prime}\right)<3 \nu$ for $i=1,2$. Hence $\frac{L}{2}=d\left(c_{1}, c_{2}\right) \leq d\left(c 1, c_{1}^{\prime}\right)+d\left(c_{1}^{\prime}, c^{\prime}\right)+d\left(c^{\prime}, c_{2}^{\prime}\right)+$ $d\left(c_{2}, c_{2}^{\prime}\right)<8 \nu$.

With little extra effort, one can prove a near-optimal bound of the same type; see Lemma 2.3.

Definition 2.2. Let $S^{1}$ be the Riemannian circle of length $2 \pi$.

Recall [5] that the filling radius of $S^{1}$ equals

(1) $\frac{1}{6}$ of its length,

(2) namely $\frac{\pi}{3}$,

(3) half the (spherical) diameter of the equilateral triangle inscribed in $S^{1}$.

For recent applications of the filling radius, see [7].

Lemma 2.3. Let $I \subseteq \mathbb{R}$ be a segment. The circle $S^{1}$ cannot be strongly isometrically embedded in a $\frac{\pi}{3}$-neighborhood of I in any metric space $Z$. 
Proof. Suppose $S^{1}$ is contained in an open $\nu$-neighborhood of $I$ in a metric space $\left(Z, d_{Z}\right)$. It will be convenient to use the language of infinitesimals, infinite proximity, etc. These terms can either be understood as shorthand for $\epsilon, \delta$ arguments, or can be interpreted literally in terms of a formalisation using an infinitesimal-enriched continuum as in [8] following [9]. We view $S^{1}$ as an infinilateral polygon. We send each vertex of the polygon to a nearest point of $I \subseteq Z$. We extend by linearity to obtain a piecewise linear map $f: S^{1} \rightarrow I$.

By the Borsuk-Ulam theorem, there is a pair of opposite points of $S^{1}$ with the same image in $I$ under $f$. Consider the infinitesimal sides $a a^{\prime} \subseteq S^{1}$ and $b b^{\prime} \subseteq S^{1}$ containing these opposite points of $S^{1}$. Then their images overlap, i.e.,

$$
f\left(a a^{\prime}\right) \cap f\left(b b^{\prime}\right) \neq \emptyset .
$$

By strong isometry and triangle inequality, we have

$$
d_{Z}(a, b) \approx \pi
$$

where $\approx$ denotes the relation of infinite proximity. Let $A A^{\prime}=f\left(a a^{\prime}\right)$ and $B B^{\prime}=f\left(b b^{\prime}\right)$. Then $\left|A A^{\prime}\right|<2 \nu$ and $\left|B B^{\prime}\right|<2 \nu$. The overlap condition (2.1) means that the segments $A A^{\prime}, B B^{\prime} \subseteq I$ overlap. Hence one of the images, say $B B^{\prime}$, must contain a vertex, say $A$, of the image $A A^{\prime}$ of the other side, so that $A \in B B^{\prime}$. We can assume that $B \in I$ is the endpoint of the side $B B^{\prime}$ closest to the point $A$. Then $d_{Z}(A, B) \leq \nu$. Thus $d_{Z}(a, b) \leq d_{Z}(a, A)+d_{Z}(A, B)+d_{Z}(B, b) \leq \nu+\nu+\nu=3 \nu$. Finally the relation (2.2) implies $\nu \geq \frac{\pi}{3}$.

\section{Separating Geodesic loop}

We choose loops $\alpha, \beta \subseteq T^{2}$ as in Section 1, and let $p=\alpha \cap \beta$. A loop in $T^{2}$ is called separating if its complement is $T^{2}$ has two connected components.

Lemma 3.1. Suppose a Riemannian torus $T^{2}$ is at Gromov-Hausdorff distance at most $\nu$ from a segment I. If $|I|>52 \nu$ then there exists an embedded separating geodesic loop $\lambda_{p}$ in $T^{2}$ based at the point $p$ (possibly non-smooth at $p$ ) and of length less than $24 \nu$.

Proof. By Lemma 1.1, the embedding of the loops $\alpha, \beta \subseteq T^{2},|\alpha| \leq|\beta|$, is strongly isometric. By Lemma 2.3 , we have $\nu \geq \frac{\pi}{3} \frac{|\beta|}{2 \pi}=\frac{|\beta|}{6}$, and therefore

$$
|\alpha| \leq|\beta| \leq 6 \nu
$$

Once we have such a "short" pair of loops on $T^{2}$, we have some indication that $T^{2}$ "looks like" a small flat torus with a Gromovian "long finger;" cf. [2, pp. 297, 321, 324, 327], [3, p. 243]. 
Suppose $T^{2}$ and $I$ are at GH distance at most $\nu$ in an ambient metric space $Z$. Choose a point $P \in I$ nearest to $p \in T^{2} \subseteq Z$. Let $Q \in I$ be an endpoint of $I$ furthest away from $P$, so that $|P Q|>26 \nu$ by hypothesis. Let $q \in T^{2}$ be a point nearest to $Q$. The point $q \in T^{2}$ can be thought of as the end of the long finger. Then

$$
\operatorname{dist}(p, q)>24 \nu
$$

Consider the commutator loop

$$
\sigma_{p}=\alpha \circ \beta \circ \alpha^{-1} \circ \beta^{-1}
$$

based at $p=\alpha \cap \beta$ as in Definition 1.4. By (3.1), the length of the commutator loop is at most

$$
\left|\sigma_{p}\right| \leq 4|\beta| \leq 24 \nu
$$

It follows from (3.2) that $q$ cannot be reached by the commutator loop $\sigma_{p}$, or by any shorter loop based at $p$.

We now cut the torus open along $\alpha$ and $\beta$. This produces a convex "parallelogram" $\Pi=\Pi_{\alpha, \beta}$. In the complement $\Pi \backslash\{q\}$, consider a shortest noncontractible loop $\lambda$ based at a corner of $\partial \Pi$. In particular, $|\lambda|<\left|\sigma_{p}\right|$. Then $\lambda$ is simple (see below), remains far away from the missing point $q$, and stays in the interior of $\Pi_{\alpha, \beta}$ (except for the basepoint) due to the convexity of $\Pi_{\alpha, \beta}$. Such a loop projects to a separating embedded loop $\lambda_{p} \subseteq T^{2}$.

To construct such a loop $\lambda$, consider the universal cover $U$ of $\Pi \backslash\{q\}$. Let $P$ be a lift of $p$ to $U$. Let $P^{\prime} \in U$ be a nearest point to $P$ in the orbit of $P$ under the action of $\pi_{1}(\Pi \backslash\{q\}, p) \simeq \mathbb{Z}$ on $U$. Let $\tilde{\lambda} \subseteq U$ by a minimizing path from $P$ to $P^{\prime}$. Let $\lambda \subseteq \Pi \backslash\{q\}$ be the geodesic loop based at $p$ obtained as the projection of $\tilde{\lambda}$. Suppose $\lambda$ is not simple. Then there is a translated path $R R^{\prime}$ meeting $\tilde{\lambda}=P P^{\prime}$ transversely at an interior point $X=P P^{\prime} \cap R R^{\prime}$. Of the 4 points $P, P^{\prime}, R, R^{\prime}$ consider the nearest one to $X$. We can assume without loss of generality that this point is $P$.

One of the points $R, R^{\prime}$ is necessarily distinct from $P$. We can assume without loss of generality that this point is $R$. Consider the broken geodesic path $P X \cup X R$. Its projection to $\Pi \backslash\{q\}$ is noncontractible, since $P \neq R$. By construction, $|P X \cup X R| \leq|\lambda|$. By rounding off the corner at $X$, we obtain a path connecting $P$ to $R$ of length strictly shorter than $|\lambda|$. This contradicts the choice of $\tilde{\lambda}$ and proves that $\lambda$ is a noncontractible simple loop of $\Pi \backslash\{q\}$.

Alternatively, one could start with the boundary $\partial \Pi$ viewed as a loop based at a corner of $\Pi_{\alpha, \beta}$, and apply Sabourau's modification [10] of 
the curve-shortening process of Hass-Scott [4]. The process preserves embeddedness and yields an embedded geodesic loop.

Note that the loop $\lambda_{p}$ of Lemma 3.1 is not necessarily a closed geodesic of $T^{2}$, as it may be nonsmooth at $p$.

\section{TORUS NON-COLLAPSE}

We now show that a 2-torus cannot collapse to a segment under the constraint $K \geq-1$.

Theorem 4.1. There is no sequence of 2-torii equipped with smooth Riemannian metrics with Gauss curvature bounded from below, which converges in the Gromov-Hausdorff sense to a (non-degenerate) segment.

Proof. Suppose there is such a sequence of metrics. For metrics sufficiently close to the segment, the hypothesis of Lemma 3.1 is satisfied. Then the loop $\lambda_{p}$ of Lemma 3.1 separates $T^{2}$ into a disk (containing $q$ ) and a torus-with-boundary $M$ in the homotopy type of $S^{1} \vee S^{1}$. The total geodesic curvature of $\lambda_{p}$ is concentrated at the point $p$ and bounded by $\pi$. We apply the Gauss-Bonnet theorem to the surface $M$ to obtain

$$
\int_{M} K d A=2 \pi \chi(M)-\int_{\lambda_{p}} k_{g}(s) d s=-2 \pi-\int_{\lambda_{p}} k_{g}(s) d s \leq-\pi .
$$

To show that the curvature has to be very negative somewhere in $M \subseteq$ $T^{2}$, it therefore suffices to show that the area of $T^{2}$ must tend to zero as the torus collapses to $I$. Indeed, consider a fine partition of $I$ with step $\nu$. For each partition point $P_{k} \in I$, choose a nearest point $p_{k} \in$ $T^{2}$. If $T^{2}$ is contained in a $\nu$-neighborhood of $I$, then by the triangle inequality, disks of radius $3 \nu$ centered at the $n=\left\lfloor\frac{|I|}{\nu}\right\rfloor$ points $p_{k}$ cover the torus:

$$
T^{2}=\cup_{k=1}^{n} B_{3 \nu}\left(p_{k}\right) .
$$

The lower bound $K \geq-1$ implies an upper bound of type $C \nu^{2}$ on the area of the disks, where $C>\pi$ can be chosen as close to $\pi$ as one wishes by choosing $\nu$ sufficiently small. It follows that the area of the torus collapsing to the segment $I$ is bounded by $\frac{|I|}{\nu} C \nu^{2}=|I| C \nu$ and hence must tend to zero with $\nu$. Then the estimate (4.1) implies that the Gaussian curvature must be very negative somewhere in $M \subseteq T^{2}$, contradicting the hypothesis of collapse of $T^{2}$ to $I$ constrained by the condition $K \geq-1$. The contradiction proves the theorem. 


\section{Klein Bottle COllapse}

Example 5.1. Unlike the torus, the Klein bottle can collapse to a segment. Let $\epsilon>0$. Consider the maps $f: \mathbb{R}^{2} \rightarrow \mathbb{R}^{2},(x, y) \mapsto(x+2, y)$ and $g: \mathbb{R}^{2} \rightarrow \mathbb{R}^{2},(x, y) \mapsto(-x, y+\epsilon)$. As a fundamental domain one can choose the rectangle $[-1,1) \times[0, \epsilon)$. Then the flat Klein bottle $K=$ $\mathbb{R}^{2} /\langle f ; g\rangle$ collapses to the segment $[0,1] \times\{0\}$ when $\epsilon \rightarrow 0$. Here the shortest basis for $H_{1}\left(K ; \mathbb{Z}_{2}\right) \simeq \mathbb{Z}_{2}^{2}$ is given by the disjoint loops parametrized by $\{0\} \times[0, \epsilon)$ and $\{1\} \times[0, \epsilon)$.

Theorem 5.2. If the Klein bottle $K$ collapses to a segment then there is a uniform positive lower bound for the minimal distance between the loops of a short basis for $H_{1}\left(K ; \mathbb{Z}_{2}\right)$.

Proof. Let $\alpha, \beta$ be such a short basis. If $K$ collapses to a segment then $|\alpha| \rightarrow 0$ and $|\beta| \rightarrow 0$ as before, based on Section 2. If there is a subsequence such that $\alpha \cap \beta \neq \emptyset$ then we cut $K$ open along $\alpha$ and $\beta$ to form a convex parallelogram $\Pi_{\alpha, \beta}$ as in the case of the torus (the identification along the boundary is different for $K$ ). We find a distant point $q \in \Pi_{\alpha, \beta}$, consider a shortest noncontractible loop $\lambda \subseteq \Pi \backslash\{q\}$ based at a corner of $\Pi_{\alpha, \beta}$, project the loop to $K$, and take the connected component $M \subseteq K \backslash \lambda$ not homeomorphic to a disk. Then $M$ is a oneholed Klein bottle in the homotopy type of $S^{1} \vee S^{1}$. We then apply the Gauss-Bonnet formula to $M$ as in (4.1), to show that collapse cannot occur in this case.

Thus we may assume that $\alpha$ and $\beta$ are disjoint. Let $\gamma$ be a shortest loop in the class $[\alpha]+[\beta] \in H_{1}\left(K ; \mathbb{Z}_{2}\right)$. Note that the class $[\gamma]$ is a characteristic vector for the $\mathbb{Z}_{2}$-intersection form, i.e., $[\gamma] \cap x=x \cap x$ for all $x \in H_{1}\left(K ; \mathbb{Z}_{2}\right)$. In particular, $\alpha \cap \gamma \neq \emptyset$.

Suppose the minimal distance between $\alpha$ and $\beta$ tends to zero. Then the length $|\gamma|$ tends to 0 , as well. We cut $K$ open along $\alpha$ and $\gamma$ to form a convex parallelogram $\Pi=\Pi_{\alpha, \gamma}$ as before. We find a distant point $q \in \Pi_{\alpha, \gamma}$, consider a shortest noncontractible loop $\lambda \subseteq \Pi \backslash\{q\}$ based at a corner of $\Pi_{\alpha, \gamma}$, take the connected component $M \subseteq K \backslash \lambda$ not homeomorphic to a disk, and apply the Gauss-Bonnet formula to $M$ as in (4.1), to show that collapse cannot occur. Therefore the minimal distance between $\alpha$ and $\beta$ cannot tend to zero.

Recently Zamora [13] generalized the non-collapsing result to tori of arbitrary dimension.

\section{ACKNOWLEDGMents}

We are grateful to Tahl Nowik, Stéphane Sabourau, and the anonymous referee for helpful comments on earlier versions of the article. 


\section{REFERENCES}

[1] Alesker, S. MathOverflow question, 2019. See https://mathoverflow.net/ q/338402

[2] Gromov, M. Systoles and intersystolic inequalities. Actes de la Table Ronde de Géométrie Différentielle (Luminy, 1992), 291-362, Sémin. Congr., 1, Soc. Math. France, Paris, 1996.

[3] Gromov, M. Metric structures for Riemannian and non-Riemannian spaces. Based on the 1981 French original With appendices by M. Katz, P. Pansu and S. Semmes. Translated from the French by Sean Michael Bates. Progress in Mathematics, 152. Birkhäuser Boston, Boston, MA, 1999.

[4] Hass, J.; Scott, P. Shortening curves on surfaces. Topology 33 (1994), no. 1, $25-43$.

[5] Katz, M. The filling radius of two-point homogeneous spaces. J. Differential Geom. 18 (1983), no. 3, 505-511.

[6] Katz, M. A quantitative obstruction to collapsing surfaces. Open Mathematics 17 (2019), 1183-1185. See https://doi.org/10.1515/math-2019-0103 and https://arxiv.org/abs/1604.06782

[7] Lim, S.; Memoli, F.; Okutan, O. Vietoris-Rips persistent homology, injective metric spaces, and the filling radius (2020), reprint. See https://arxiv.org/ abs/2001.07588

[8] Nowik, T; Katz, M. Differential geometry via infinitesimal displacements. Journal of Logic and Analysis 7:5 (2015), 1-44. See http://www. logicandanalysis.com/index.php/jla/article/view/237 and https:// arxiv.org/abs/1405.0984

[9] Robinson, A. Non-standard analysis. North-Holland Publishing, Amsterdam 1966.

[10] Sabourau, S. Filling radius and short closed geodesics of the 2-sphere. Bull. Soc. Math. France 132 (2004), no. 1, 105-136.

[11] Shioya, T.; Yamaguchi, T. Collapsing three-manifolds under a lower curvature bound. J. Differential Geom. 56 (2000), no. 1, 1-66.

[12] Yamaguchi, T. Collapsing and pinching under a lower curvature bound. Ann. of Math. (2) 133 (1991), no. 2, 317-357.

[13] Zamora, S. Tori can't collapse to an interval. Preprint (2020). See https:// arxiv.org/abs/2004.01505v3

M. Katz, Department of Mathematics, Bar Ilan University, Ramat GAN 52900 ISRAEL

E-mail address: katzmik@macs.biu.ac.il 